an die Anerkennung von Kursen im Sinne des $₫ 18$ a Abs. 4 RöV gestellt werden dürfen.

Die ablehnende Entscheidung stellt keine Verletzung der Berufsausübungsfreiheit (Art. 12 Abs. 1 GG) der Kl. dar. Soweit in der Verlängerung der Präsenzphase um etwa dreieinhalb Stunden bei einer Kursdauer von insgesamt 18 Stunden überhaupt ein relevanter Grundrechtseingriff zu sehen ist, ist dieser jedenfalls durch den Gemeinwohlbelang gerechtfertigt, die Gesundheit von Patienten und Mitarbeitern vor Strahlen zu schützen, die vom Betrieb von Röntgeneinrichtungen ausgehen.

2. Die Berufung ist nicht wegen der geltend gemachten Divergenz ( $(124$ Abs. 2 Nr. 4 VwGO) zuzulassen. Die Kl. trägt dazu vor, das VG habe den abstrakten Rechtssatz aufgestellt, (wird ausgeführt) [...]

3. Die Berufung ist ferner nicht wegen der geltend gemachten grundsätzlichen Bedeutung der Rechtssache nach $\int 124$ Abs. 2 Nr. 3 VwGO zuzulassen. Eine Rechtssache hat grundsätzliche Bedeutung im Sinne dieser Vorschrift, wenn sie eine konkrete noch nicht geklärte Rechts- oder Tatsachenfrage aufwirft, deren Beantwortung sowohl für die Entscheidung des VG von Bedeutung war als auch für die Entscheidung im Berufungsverfahren erheblich sein wird und die über den konkreten Fall hinaus wesentliche Bedeutung für die einheitliche Anwendung oder für die Weiterentwicklung des Rechts hat. (wird ausgeführt) [...]

https://doi.org/10.1007/s00350-019-5382-9

\section{Anmerkung zu OVG Nordrhein-Westfalen, Beschl. v. 21.9.2018 - 13 A 300/17 (VG Köln) Hans-Dieter Lippert}

Die vorstehend abgedruckte Entscheidung des OVG Nordrhein-Westfalen ist zu \18a RöV in der Fassung von 1987 ergangen. Wer sie sucht, wird sich schwer tun, sie noch zu finden, wohl am ehesten noch im Bundesgesetzblatt. Denn die Umsetzung der Richtlinie 2013/59/Euratom ${ }^{1}$ hat $\mathrm{zu}$ einer umfänglichen Neuregelung des gesamten Rechts des Strahlenschutzes geführt. Bisher lieb gewordene Zitate von Paragraphen aus der StrlSchVO ${ }^{2}$ aber auch der RöV ${ }^{3}$ führen in die Leere, weil diese Verordnungen am 31.12.2018 außer Kraft getreten sind. Ihre Stelle eingenommen haben u. a. das StrlSchG ${ }^{4}$ und die Verordnung zur weiteren Modernisierung des Strahlenschutzes ${ }^{5}$. In einer Vielzahl weiterer Gesetze und Rechtsverordnungen hat dies zu größeren und kleineren Veränderungen geführt.

Insgesamt ist zu den neuen Regelungen zu sagen, dass damit der Strahlenschutz nicht neu erfunden wird. Es wird mit den Vorschriften aber auf einen technisch aktuellen Stand gebracht. Dass dadurch der Umfang der Vorschriften zugenommen hat überrascht nicht wirklich. Es entspricht dies dem Lauf der Zeit. Einfacher wird durch eine Neuregelung nichts.

Doch zurück zu unserem Ausgangsfall. Der Inhalt von S18a RöV 1987 ist keinesfalls ersatzlos untergegangen. Wer sich künftig im Strahlenschutz tummeln möchte, der tut gut daran, sich möglichst schnell mit den neuen Normen anzufreunden. Auch das StrlSchG geht in Kapitel 4, welches die betriebliche Organisation des Strahlenschutzes regelt, in $\$ 74$ davon aus, dass nur Personal mit der erforderlichen Fachkunde im Strahlenschutz durch geeignete Einweisung und praktische Erfahrung tätig werden darf. Die Einzelheiten dafür finden sich in der StrlSchVO ${ }^{6}$. In $\$ 47$ StrlSchVO ist geregelt, wie diese Fachkunde erworben werden kann, nämlich

Rechtsanwalt Dr. iur. Hans-Dieter Lippert,

KNORR Rechtsanwälte AG,

Frauenstr. 11, $89073 \mathrm{Ulm}$, Deutschland durch eine für das jeweilige Anwendungsgebiet geeignete Ausbildung, durch den Nachweis praktischer Erfahrung und schlussendlich durch die erfolgreiche Teilnahme an anerkannten Kursen. An diesem bisher bereits erfolgreich praktizierten System hält auch die neue StrlSchVO fest. Eine Anerkennung von Kursen, die bisher nach $\$ 18$ a RöV Grundlage für die Anerkennung von Kursen war, regelt nun $\$ 51$ StrlSchVO. Die Anforderungen unterscheiden sich dabei nicht von denjenigen, die nach $\$ 18 \mathrm{a}$ RöV zu erbringen waren.

Ein kleiner Exkurs: auch diejenigen Personen, die in der biomedizinischen Forschung tätig werden und ionisierende Strahlen anwenden wollen, müssen sich auf die neuen Vorschriften einstellen. An die Stelle der \$28 RöV und $\$ 91$ StrlSchVO sind zum 1.1.2019 die $\$ \S 31$ ff. StrlSchG und $\int \$ 133$ StrlSchVO getreten. Für Forschungsprojekte, die durch eine Ethikkommission bewertet werden müssen, gilt $\$ 36$ StrlSchG.

1) Richtlinie 2013/59/Euratom des Rates v. 5.12.2013 zur Festlegung grundlegender Sicherheitsnormen für den Schutz vor den Gefahren einer Exposition gegenüber ionisierender Strahlung und zur Aufhebung der Richtlinien 89/618/Euratom, 90/641/Euratom, 96/29/Euratom, 97/43/Euratom und 2003/122/Euratom (AB1., L13, v. 17. 1.2014, S. 1.).

2) Strahlenschutzverordnung v. 20.7.2001 (BGB1. I S. 1714; 2002 I S. 1459), die zuletzt durch Art. 6 des Gesetzes v. 27.1.2017 (BGB1. I S. 114, 1222, 1676) geändert worden ist. (außer Kraft).

3) Röntgenverordnung i.d.F.d. Bekanntmachung v. 30.4.2003 (BGB1. I S. 604), die zuletzt durch Art. 6 der Verordnung v. 11.12.2014 (BGBl. I S. 2010) geändert worden ist. (außer Kraft).

4) Gesetz zum Schutz vor der schädlichen Wirkung ionisierender Strahlung (Strahlenschutzgesetz - StrlSchG) StrlSchG v. 27.6.2017 (BGBl. I S. 1966), das durch Art. 2 des Gesetzes v. 27.6.2017 (BGBl. I S. 1966) geändert worden ist.

5) Verordnung zur weiteren Modernisierung des Strahlenschutzrechts (StrlSchModV) Verordnung v. 29.11.2018 (BGB1. I S. 2034). Die Art. 1 bis 3, 5 bis 14 und 16 bis 20 dieser Verordnung dienen der Umsetzung der Richtlinie 2013/59/Euratom des Rates v. 5.12.2013 zur Festlegung grundlegender Sicherheitsnormen für den Schutz vor den Gefahren einer Exposition gegenüber ionisierender Strahlung und zur Aufhebung der Richtlinien 89/618/ Euratom, 90/641/Euratom, 96/29/Euratom, 97/43/Euratom und 2003/122/Euratom (ABl. L 13 v. 17.1.2014, S. 1). Die Art. 1 und 18 dieser Verordnung dienen auch der Umsetzung des Art. 8d der Richtlinie 2009/71/Euratom des Rates v. 25.6.2009 über einen Gemeinschaftsrahmen für die nukleare Sicherheit kerntechnischer Anlagen (ABl. L 172 v. 2.7.2009, S. 18), in der durch die Richtlinie des Rates 2014/87/Euratom v. 8.7.2014 zur Änderung der Richtlinie 2009/71/Euratom über einen Gemeinschaftsrahmen für die nukleare Sicherheit kerntechnischer Anlagen (ABl. L 219 v. 25. 7.2014, S. 42) geänderten Fassung.

6) Verordnung zum Schutz vor der schädlichen Wirkung ionisierender Strahlung (Strahlenschutzverordnung - StrlSchVO) v. 29.11.2018 (BGB1. I S. 2034, 2036).

\section{Ausschreibung eines hälftigen Vertragsarztsitzes bei Weiternutzung der anderen Hälfte - Aufteilung der Fallzahlen}

GG Art. 12 i. V. mit Art. 3; SGB V §§87b Abs. 1, Abs. 2 S. 1, 95 Abs. 3 S. 1; HVM 2013 der KVBW

1. Bei der Ausschreibung eines hälftigen Vertragsarztsitzes unter Weiternutzung der anderen Hälfte wird die RLV relevante Fallzahl des Ausschreibenden nicht

Eingesandt von Prof. Dr. iur. Thomas Clemens, Kassel; bearbeitet von Rechtsanwalt Dr. iur. Felix Heimann, Rechtsanwälte M\&P Dr. Matzen und Partner mbB, Neuer Wall 55, 20354 Hamburg, Deutschland 